\title{
Spectroscopy-on-chip applications of silicon photonics
}

\author{
Roel Baets, Ananth Z. Subramanian, Ashim Dhakal, Shankar K. Selvaraja, Katarzyna Komorowska, \\ Frédéric Peyskens, Eva Ryckeboer, Nebiyu Yebo, Gunther Roelkens and Nicolas Le Thomas \\ Photonics Research Group, INTEC-department, Ghent University - imec \\ Center for Nano- and Biophotonics, Ghent University \\ Sint-Pietersnieuwstraat 41, B-9000 Ghent, Belgium
}

\begin{abstract}
In recent years silicon photonics has become a mature technology enabling the integration of a variety of optical and optoelectronic functions by means of advanced CMOS technology. While most efforts in this field have gone to telecom and datacom/interconnect applications, there is a rapidly growing interest in using the same technology for sensing applications, ranging from refractive index sensing to spectroscopic sensing. In this paper the prospect of silicon photonics for absorption, fluorescence and Raman spectroscopy on-a-chip will be discussed. To allow spectroscopy in the visible and near infrared the silicon photonics platform is extended with silicon nitride waveguides.
\end{abstract}

Keywords: Integrated photonics, absorption spectroscopy, fluorescence, Raman spectroscopy, silicon, silicon-nitride

\section{INTRODUCTION}

Optical spectroscopy is a key technology for the detection of a wide variety of atoms and molecules in applications ranging from physics and chemistry to biology and environmental sciences. As compared to many other types of sensors spectroscopic techniques have the advantage that their selectivity stems from the "fingerprint" of the spectrum rather than from chemical affinity of a given molecule to a functionalized surface. Absorption spectroscopy, fluorescence spectroscopy and Raman spectroscopy all provide complementary information about the identity and concentration of the molecules. In spite of these advantages spectroscopic techniques often require bulky or expensive instrumentation. If key parts of the optical functionality could be integrated in the form of a chip, the route would be opened to miniaturized low cost systems, with the potential of massive parallelism and multiplexing [1,2].

One photonic integration technology that has reached considerable maturity in recent years is silicon photonics. This technology makes use of the technologies available in an advanced CMOS fab to fabricate photonic integrated circuits on 200 or $300 \mathrm{~mm}$ silicon-on-insulator (SOI) wafers with passive waveguide circuits, optical modulators and detectors, thermo-optic phase tuners and - when combined with bonded III-V semiconductor layers - light sources, lasers in particular [3-6]. Since silicon is only transparent above $1.1 \mu \mathrm{m}$, the technology is not readily suitable for applications requiring shorter wavelengths, as is often the case in biology. But by making use of silicon-nitride $\left(\mathrm{Si}_{3} \mathrm{~N}_{4}\right)$ waveguide layers instead of silicon, the technology can be extended to the entire visible and near infrared wavelength range. This is particularly relevant for Raman spectroscopy and fluorescence spectroscopy. In the case of vibrational absorption spectroscopy it is beneficial to work at mid-infrared wavelengths where the absorption is strong. This calls for the replacement of silicon-oxide by a cladding material that is transparent in the mid IR.

The asset of using optical chips goes beyond the cost and reliability advantage associated with the use of mature waferscale technologies and the miniaturization per se. There are also performance advantages. One important element is the fact that the high refractive index contrast in an SOI waveguide and, to a lesser extent, in a $\mathrm{Si}_{3} \mathrm{~N}_{4}$ waveguide helps to enhance the electric field strength of a guided mode for a given mode power. This is beneficial both for the absorption rate of the guided mode by a particle seen by the enhanced field and for the enhancement and coupling of light emitted by a particle coupled to the guided mode. This enhancement can be boosted further through either resonant cavity

Integrated Optics: Devices, Materials, and Technologies XVII, edited by Jean Emmanuel Broquin,

Gualtiero Nunzi Conti, Proc. of SPIE Vol. 8627, 86270I - (c) 2013 SPIE

CCC code: $0277-786 \mathrm{X} / 13 / \$ 18 \cdot$ doi: $10.1117 / 12.2010498$

Proc. of SPIE Vol. $8627862701-1$ 
enhancement and/or plasmonic enhancement by means of metallic nanostructures in the vicinity of the waveguides. Furthermore light coupled to a single waveguide mode has a perfect spatial coherence and can in principle be decomposed in its spectral components with unlimited resolution.

In spite of this promise there also remain enormous challenges. The relevant signals both in absorption and emission spectroscopy are often extremely weak. Therefore, apart from enhancement mechanisms there is a need for detection systems with very low noise and very low drift. In the case of emission spectroscopy there is a need for high performance filters to reject the pump wavelength. Auto-fluorescence of the waveguide materials is another source of crosstalk that may need mitigation.

In this paper a number of theoretical and technological elements will be touched upon that are relevant to make the dream of spectroscopy-on-a-chip come true. In section 2 the enhancement of emission and absorption in high index contrast (HIC) waveguides is discussed. Section 3 touches upon the combination of waveguide confinement and plasmonic enhancement, which is especially relevant for spontaneous Raman spectroscopy. In section 4 examples are given of chips that perform absorption spectroscopy of fluids and gases on a chip. Finally section 5 discusses the development of a $\mathrm{Si}_{3} \mathrm{~N}_{4}$ waveguide platform, with focus on the development of surface normal light coupling to the chip by means of grating couplers.

\section{WAVEGUIDE ENHANCEMENT OF EMISSION AND ABSORPTION}

\subsection{Waveguide Enhancement of emission}

A significant amount of emission, from a particle sufficiently close to a waveguide core, can get coupled into the guided modes of the waveguide [7-11]. In certain conditions, this amount can be larger than it would be in free space in all directions. This is possible due to Purcell enhancement [12] near the waveguide and optical confinement.

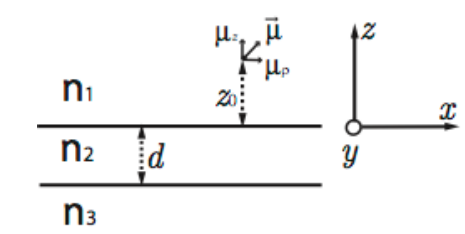

a) b)

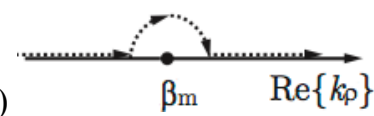

Figure 1. a) The three layered slab waveguide under discussion. Core slab of thickness $d$ is sandwiched in between semi-infinite upper cladding and lower substrate of lower refractive indices. The dipole is located in the upper cladding at a height $z_{0}$ from the surface of the core at $\boldsymbol{r}_{0}=\left(x_{0}, y_{0}, z_{0}\right)$. b) The integration path to evaluate Eq. (2) in the presence of a guided mode. Integration around the semicircle of infinitesimal radius at the pole location corresponding to the guided mode gives the power coupled to the mode.

To simplify the emission problem near waveguides, the emitter can be modeled as a classical electric dipole of strength, $\boldsymbol{\mu}$, oscillating at a frequency, $\omega$, near a slab waveguide of thickness $d$, as depicted in Fig. 1 a). Poynting's theorem for a current distribution dictates that the emitted power of a dipole is dependent on the field in the direction of the dipole moment and at the location of the dipole $\boldsymbol{r}_{0}$. The total field is the superposition of the primary dipole field, $\boldsymbol{E}_{\mathbf{0}}\left(\boldsymbol{r}_{0}\right)$ and the secondary field $E_{\mathrm{s}}\left(\boldsymbol{r}_{0}\right)$ of the dipole scattered by the waveguide, hence the total power radiated can be written as [9],

$$
\mathrm{P}=\frac{\omega}{2} \operatorname{Im}\left[\vec{\mu} \cdot\left(\overrightarrow{\mathrm{E}}_{0}\left(\mathrm{r}_{0}\right)+\overrightarrow{\mathrm{E}}_{\mathrm{s}}\left(\overrightarrow{\mathrm{r}}_{0}\right)\right)\right]
$$

This means that the scattered field, $\boldsymbol{E}_{\mathbf{s}}\left(\boldsymbol{r}_{\boldsymbol{0}}\right)$ governs the modification of the emission rate of the dipole. One well-known method to evaluate Eq. (1) is to expand the dipole field into its plane wave components and to apply general reflection formulae to evaluate the field at the location of the dipole. With this method, the total emission by the dipole, normalized to the free space emission, can be calculated to be [8-9] 


$$
\frac{P}{P_{0}}=1+\operatorname{Re} \int_{0}^{\infty} d k_{\rho} \frac{\mu_{\rho}^{2}}{\mu^{2}} \frac{3}{4} \frac{k_{\rho}}{k_{1}^{3} k_{1 z}}\left(k_{1}^{2} r_{T E}-k_{1 z}^{2} r_{T M}\right)+\frac{\mu_{z}^{2}}{\mu^{2}} \frac{3}{2} \frac{1}{k_{1}^{3} k_{1 z}}\left(k_{\rho}^{3} r_{T M}\right)
$$

Where, $r_{T E}$ and $r_{T M}$ are the reflectivities of the total structure for TE and TM polarizations; $k_{l}$ and $\mu$ are respectively the wave number in the upper cladding and the total dipole moment, $\mu_{\rho}$ and $k_{\rho}$, are the corresponding horizontal components and $\mu_{z}$ and $k_{l z}$ are the corresponding vertical components. It is well known [8-10] that the guided modes correspond to the poles of the total reflectivities, $r_{T E}$ and $r_{T M}$ of the structure. The power coupled to the mode or the modal contribution to the total emission of the dipole for a given guided mode can be calculated by integrating Eq. 3 along the infinitesimal contour around the corresponding poles (see Fig. $1 \mathrm{~b}$ ).

Using Gauss-Kronrod quadrature algorithm [13], one can perform the complex integration around the poles numerically. In Fig. 2, we show the power $P_{m}$ coupled to the fundamental TE and TM guided modes as a function of the core thickness, for $\mathrm{Si}$ and $\mathrm{Si}_{3} \mathrm{~N}_{4}$ cores on a silica substrate and air cladding for the two distinct dipole orientations. It is clear that as much as 5 times and 1.6 times the power radiated in the free space can be coupled to the simple slab waveguides with silicon and $\mathrm{Si}_{3} \mathrm{~N}_{4}$ cores respectively. The power coupled is dependent on the core thickness, polarization of the mode and the orientation of the dipole, and follows roughly the modal field intensity at the dipole location $\left|\boldsymbol{E}_{\boldsymbol{m}}\left(\boldsymbol{r}_{\boldsymbol{O}}\right)\right|^{2}$.
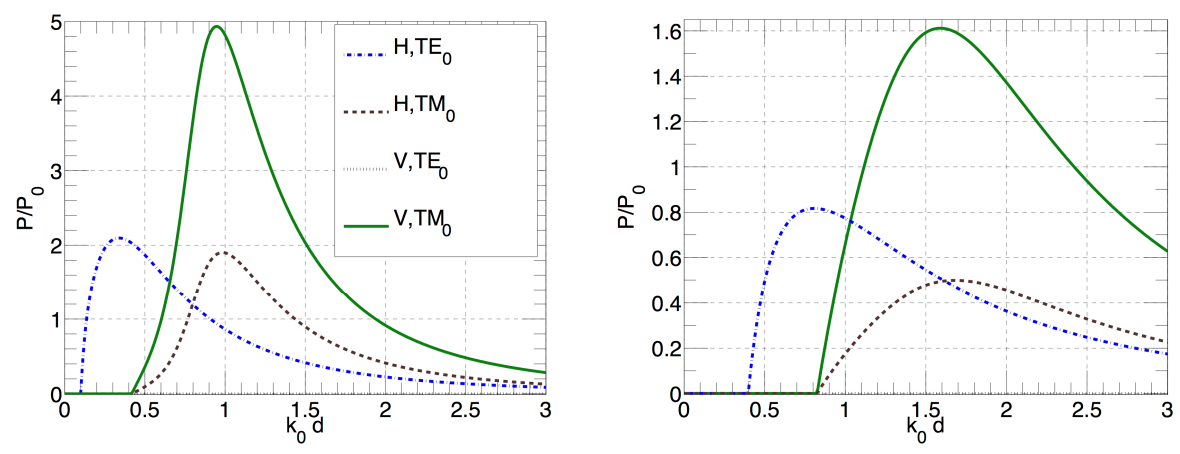

Figure 2. The power coupled to the fundamental TE and TM modes of a slab waveguide normalized to total free space emission in all the directions, for $\mathrm{Si}$ (left) and $\mathrm{Si}_{3} \mathrm{~N}_{4}$ (right) cores on silica substrate and air cladding. The powers are calculated for dipoles on the core surface and oriented vertical $(\mathrm{V})$ and horizontal $(\mathrm{H})$ to it.

\subsection{Overall efficiency of absorption and emission from a guided mode}

It is well known that the absorption by a particle is proportional to the field intensity at the location of the particle. In the presence of a guided mode and no other source of field at resonance, the absorption is proportional to the modal field intensity at the location of the dipole, $\left|\boldsymbol{E}_{\boldsymbol{m}}\left(\boldsymbol{r}_{0}\right)\right|^{2}$. If we neglect stokes shift, and assume that both the dipole emission and absorption are at resonance with the waveguide field, the overall efficiency of absorption and subsequent emission can be calculated as the product of absorption and emission rates. In Fig. 3, we calculate the overall efficiencies for all the possible pumping-coupling combinations for the fundamental modes, (i.e. $\mathrm{TE}_{0}-\mathrm{TE}_{0}, \mathrm{TE}_{0}-\mathrm{TM}_{0}, \mathrm{TM}_{0}-\mathrm{TE}_{0}, \mathrm{TM}_{0}-\mathrm{TM}_{0}$ ) and random dipole orientation, i.e., owing to the horizontal invariance of the problem; one third of emitted power is considered due to vertical dipole and the rest due to horizontal components. We see that the TM pumped-TM coupled scheme yields the best overall efficiency at the optimal width. It is also found that the dependence of this total efficiency on index contrast is roughly quadratic [7]. 

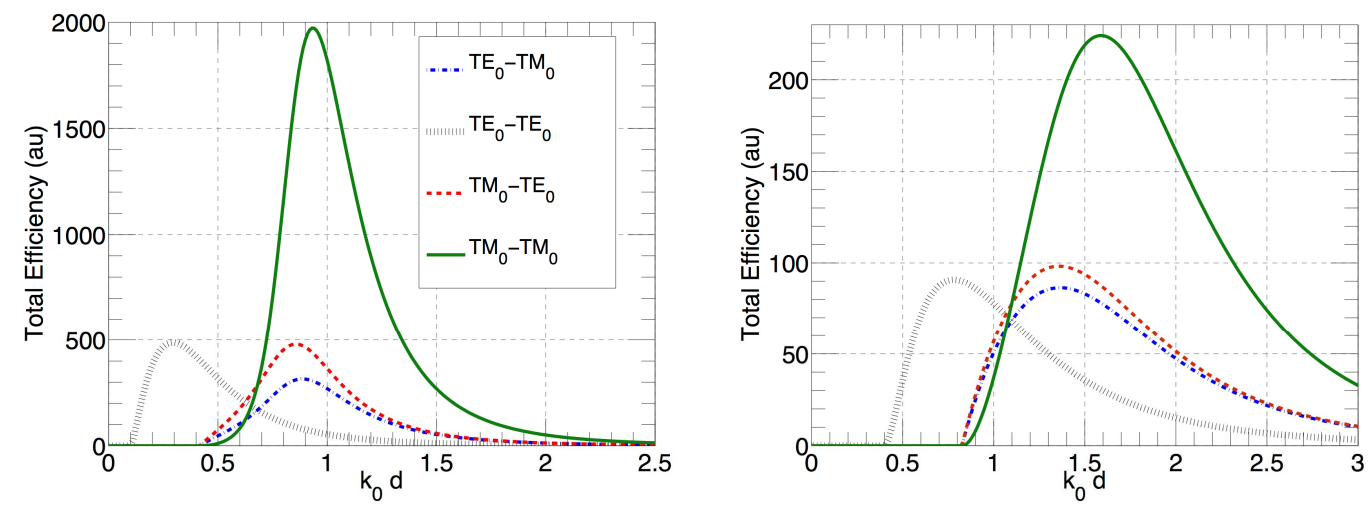

Figure 3. The overall efficiency for all possible pump-coupling possibilities (e.g. $\mathrm{TE}_{0}-\mathrm{TM}_{0}$ meaning that dipole is excited with $\mathrm{TE}_{0}$ and the emission is coupled to $\mathrm{TM}_{0}$ ).

\section{PLASMONIC ENHANCEMENT OF ABSORPTION/EMISSION}

\subsection{The potential of localized surface plasmons for spectroscopic sensing}

The use of plasmonics for spectroscopic applications stems from the enhanced fields associated to their plasmon resonance. One can distinguish between the Surface Plasmon Polaritons (SPPs) and the Localized Surface Plasmons (LSPs) [14]. SPPs are propagating plasmons at the interface between a metal and a dielectric. LSPs are localized plasmons at the tips or edges of metallic nanostructures. In both cases the electric field at the metal-dielectric interface is enhanced. This intensity increase is used to boost spectroscopic Raman signals which are inherently very weak [15]. SPPs extend further into the dielectric $(\sim 150 \mathrm{~nm})$ compared to LSPs, which are typically tightly confined to the surface $(\sim 25 \mathrm{~nm})$. In that sense the SPPs can probe further into the material that is deposited at the interface. However, the field enhancement that can be achieved with SPPs is generally much smaller (10-100 times) than that obtained from the LSPs. For probing nano-size particles (such as molecules), which cannot benefit from the larger probing region, stronger fields are more beneficial to enhance the very weak Raman signals they emit. Hence, the LSPs appear to be better candidate for probing such small particles.

\subsection{Plasmonic nanostructures integrated on SiN waveguides}

LSPs are very sensitive to the polarization and wavelength of the exciting beam, the dielectric environment and the size and shape of the specific nanostructure, so the design of proper nanostructures integrated on HIC waveguide platform is therefore of critical importance when one envisages on-chip Raman spectroscopy [16]. The induced Raman-dipole is given by $\mu=\alpha_{\text {Raman }} \cdot E_{\text {loc }}$, where $\alpha_{\text {Raman }}$ is the Raman tensor and $E_{\text {loc }}$ is the local electric field. The local field is enhanced with respect to the incident evanescent field $E_{\text {in }}$ of the waveguide $\left(E_{\text {loc }}=\beta E_{\text {in }}, \beta>1\right)$. In many cases it is correct in good approximation that the emitted Raman Stokes light undergoes the same enhancement mechanism. This implies that both the local incident intensity on the molecule $\mathrm{P}_{\text {loc }} \sim \beta^{2} \mathrm{P}_{\text {in }}$ (where $\mathrm{P}_{\text {in }}$ is the incident intensity on the nanostructure) and the enhanced emitted Raman intensity $\mathrm{P}_{\mathrm{ER}} \sim \beta^{2} \mathrm{P}_{\text {loc }}$ scale with $\beta^{2}$. In that sense the Raman enhancement factor (EF) is given by $\beta^{4}$. In reality enhancement of the emission will depend on the orientation of the dipole ([17]) but the approximation described above is relevant for excitation through the fundamental TE-mode of a waveguide. In this case the dominant component of the excitation field will polarize the dipole along the axis parallel to the TE-mode, hence the oscillatory motion of the dipole is also along the main axis of the metallic nanostructure (see inset Figure 4 (right)). This will produce a similar plasmon resonance as for the excitation case. Figure 4 (left) shows the EF of a triangle dipole as a function of the length offset. The optimal configuration is for an offset value of zero. A deviation of only $+40 \mathrm{~nm}$ reduces the EF with a factor of 100 and increasing the gap with $30 \mathrm{~nm}$ gives a reduction by a factor of 1000 . Higher EFs $\left(10^{10}\right)$ can be achieved by tuning the complete geometry parameter space. In Figure 4 (right) the LSP for an on-chip 
dipole of triangles is plotted. The tightly confined mode, closely packing the field around the tips, is clearly visible and can be used for detecting Raman signals of molecules or nanoparticles that are situated in the gap region.
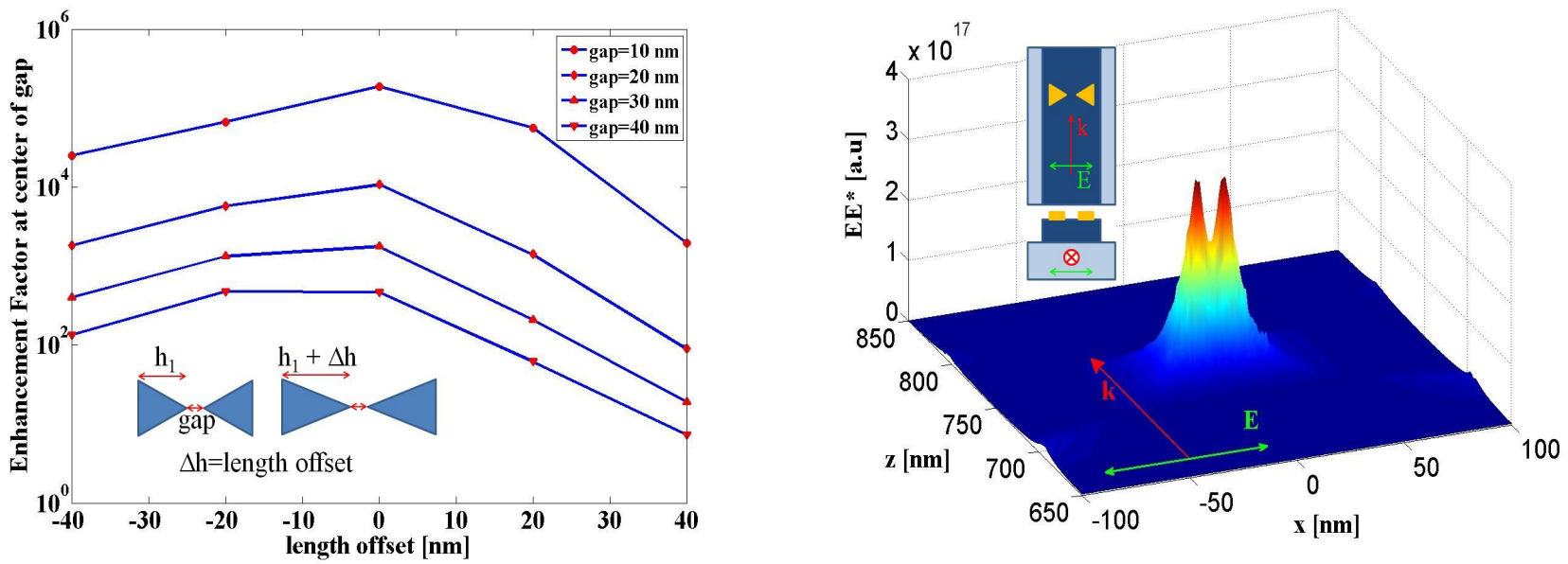

Figure 4. Left: Enhancement factor as a function of size parameters; Right: LSP for a dipole of triangles excited by the evanescent field (wave-vector and polarization are shown in red and green respectively) of a $\mathrm{Si}_{3} \mathrm{~N}_{4}$ rib waveguide (inset figure).

Using an integrated waveguide $\left(\mathrm{Si}_{3} \mathrm{~N}_{4}\right.$ or $\mathrm{Si}$ )-plasmonics platform also offers a great advantage since LSPs are strongly dependent on the incident polarization of the electric field. The metallic structures can be fabricated such that they are always properly aligned with the mode-polarization of the waveguide. This maximizes the excitation efficiency of the plasmons with respect to randomly oriented gold clusters used in more conventional Surface Enhanced Raman Spectroscopy (SERS) schemes.

\section{ABSORPTION SPECTROSCOPY ON A SOI CHIP}

\subsection{Dealing with the short interaction length on a chip}

Every molecule has a unique infrared absorption spectrum that stems from its molecular vibrations and rotations. The absorption is strongest at the fundamental vibration frequencies, but these are mostly inaccessible with the present SOI platform. However, as the molecules can be represented as inharmonic oscillators, overtone transitions occur, that are weaker in nature but can be detected in the near-infrared. For example, the first overtones of $\mathrm{C}-\mathrm{H}$ stretching vibrations can be accessed in the wavelength range of $1530-1820 \mathrm{~nm}$. To probe a molecule with an optical waveguide, the evanescent field of the optical mode in the cladding containing the molecule is exploited. This field remains confined to the core-cladding interface with a typical 1/e decay length of $60 \mathrm{~nm}$ (for $\lambda=1.56 \mu \mathrm{m}$ ) in a $200 \mathrm{~nm}$ high and $500 \mathrm{~nm}$ wide silicon ridge waveguide with water cladding [18]. The evanescent field is attenuated due to absorption in the cladding. This process is well described by the law of Beer-Lambert $T=\exp \left(-\left(\Gamma \alpha_{s}+\alpha_{w g}\right) L=10^{-4}\right.$. This law relates the waveguide transmission $T$, after a distance of $L$, with the losses in both waveguide $\left(\alpha_{\mathrm{wg}}\right)$ and cladding $\left(\alpha_{\mathrm{s}}\right)$. The definition of absorbance $A$, expressed in absorbance units (A.U.), is also included. The fill factor $\Gamma$ is the fraction of the optical mode that extends in the cladding. As this factor is a relatively small number for ridge waveguides, typically 0.1, a long distance $\mathrm{L}$ is required. To reduce the sensor footprint, while maintaining a long interaction length $\mathrm{L}$, the sensing waveguide can simply be routed into a spiral shape. It is possible to determine an optimal interaction length for a given fill factor and waveguide losses. Key is to optimize the power difference between the measurement with and without sample absorption. If the interaction length is chosen too short, the absorption features are not evident, whereas a longer length decreases the detected power too strongly. The optimal interaction length $\mathrm{L}_{\text {opt }}$ is given by $L_{o p t}=1 /\left(\Gamma \alpha_{s}+\alpha_{w g}\right)$. In other words the power at the end of the waveguide should be 1/e times the power at the input. One should keep in mind, however, that this length is wavelength dependent. This equation also indicates that a low-loss waveguide in combination 
with a larger fill factor $\Gamma$ is advantageous. Other sensing configurations such as cavity enhanced sensors increase the interaction length by increasing the Q-factor of the cavity [19]. It is also possible to obtain a sufficient interaction length by using slow-light which enhances the light-matter interaction [20].

In the case of gas absorption spectroscopy, very long interaction lengths are required to obtain a strong signal due to the very low molecular density. Physically adsorbing films on optical structures can boost the number density of gas molecules near the interaction region. High surface area, homogenous films of organic or inorganic materials can be considered for this purpose. Poly-dimethylsiloxane (PDMS) is one of the attractive materials as a gas pre-concentrating layer. Our preliminary results indicate that this material can efficiently concentrate organic vapors such as xylene.

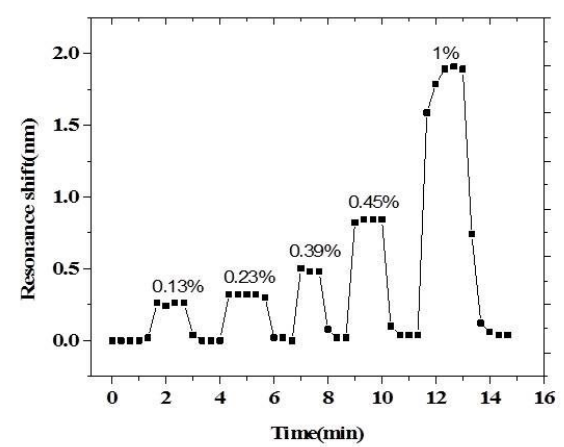

Figure 5. Resonance shift measured from a PDMS functionalized silicon micro-ring resonator with different xylene vapor concentrations.

Figure 5 shows the response of a microring resonator coated with $\sim 150 \mathrm{~nm}$ thick PDMS upon exposure to different xylene vapor concentrations. Refractive index changes over $1 \times 10^{-2}$ are estimated for concentrations below $1 \%$ of xylene in nitrogen. This allowed us to estimate that two to three orders increase in the density of the gas molecules is possible by using such a pre-concentration scheme. If effectively implemented for waveguide based absorption spectroscopy, this technique can potentially compensate for the short interaction length on optical chips. Moreover, the highly reversible response of the PDMS coating indicates the physical nature of xylene adsorption, which may have a key advantage to keep the spectral features of the molecules hardly distorted by the adsorption process. The fast and reversible response is further attractive as it can lead to reusable sensors for real time applications.

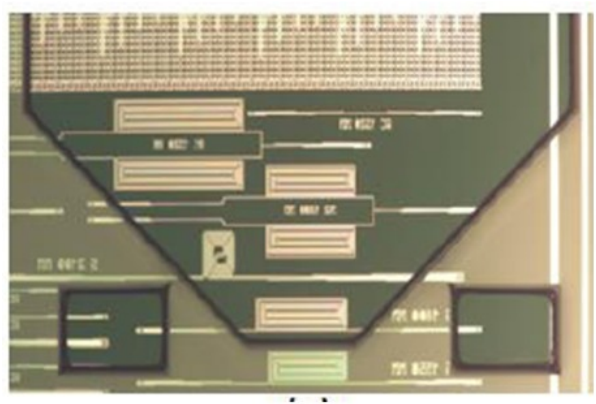

(a)

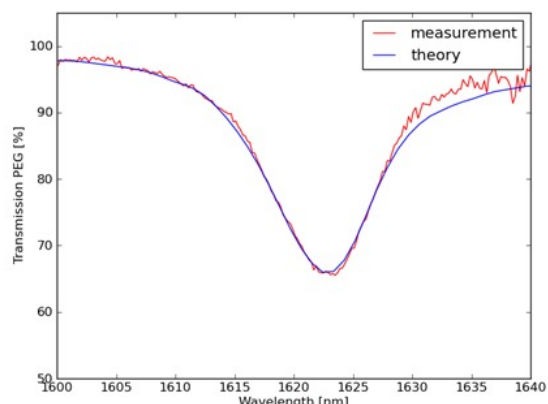

(b)

Figure 6. (a) Microscope image of single-beam optical chip (b) measured spectrum of PEG

\subsection{Absorption spectroscopy with a broadband SLED}

A straightforward set-up for on-chip absorption spectroscopy is a single-beam configuration using a SOI spiral waveguide. Here we present results for a spiral waveguide with a length of $1 \mathrm{~cm}$. The waveguide is a $220 \mathrm{~nm}$ thick and $450 \mathrm{~nm}$ wide silicon ridge with a shallow etch depth of $70 \mathrm{~nm}$ on a $2 \mu \mathrm{m}$ thick buried oxide layer. Figure 6.a. shows a 
microscope picture of the optical chip. The chip is covered with a $50 \mu \mathrm{m}$ thick SU8 layer except for a sensing window above the spiral and openings above the grating couplers to fill with glue. UV-curable glue is applied in these openings to fix the optical fibers to the optical chip. A super-luminescent LED (SLED) is combined with an optical spectrum analyzer for read-out. An example measurement is shown in figure 6.b. The theoretical curve is based on an FTIR measurement and a fitted confinement factor of 0.131 . The spiral is completely covered by a pure poly-ethylene glycol (PEG) solution. This liquid polymer has a clear absorption dip at $1620 \mathrm{~nm}$. The short-time stability of the system is characterized by calculating the root mean square (RMS) noise for $100 \%$ lines. The $100 \%$ lines are computed by dividing replicate spectra. With this set-up, the RMS noise amounts to $544 \mu \mathrm{AU}$ over a $20 \mathrm{~nm}$ wide spectral window where the detected power is maximum. With appropriate averaging strategies, a signal-to-noise ratio (SNR) of 1500 can be obtained before slow drift effects take over. In this single-beam type of configuration, we are ultimately limited by the source power and its fluctuations. One way to increase the SNR is to use a dual-beam set-up. In this type of set-up the source light is split into two paths. One path is a reference path and the second path is the signal path that contains the sample under test. All fluctuations common to both reference and signal, as well as source fluctuations, can thus be removed. For a broadband source this requires, however, two optical spectrum analyzers, or an optical switch, which might introduce non-common noise. By changing to a laser source with tunable wavelength, this issue is alleviated.

\subsection{Laser absorption spectroscopy}

In figure 7 (a) an example dual-beam circuit is shown. To achieve simultaneous and stable input and read-out, a fiber array is glued to the optical chip. In addition, micro-fluidics in PDMS is used to allow easy transfer of fluids to and from the chip. The circuit contains two identical reference spirals and two identical signal spirals and all four are accessed by the same input source. To show the advantage of a dual-beam vs. a single-beam set-up, we calculated the SNR of both the signal and reference and their ratio. The dual-beam approach improves the SNR by a factor of five over a wide spectral range. While a laser source offers a SNR advantage in terms of enhanced signal strength, the use of coherent light in combination with an integrated optical chip has one significant disadvantage. An integrated SOI chip is prone to interference effects caused by sidewall roughness and unwanted optical cavities. Slight deviations in lasing wavelength stability during consecutive scans can be converted into large power variations with a spectral signature over a large wavelength range. The use of optimized components such as reflection-less grating couplers [21], low-reflection multimode interference splitters (MMI) [22] and spirals with spline bends [23] reduce the impact of this phenomenon.

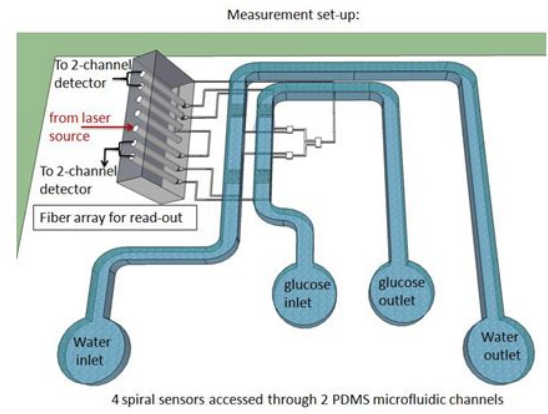

(a)

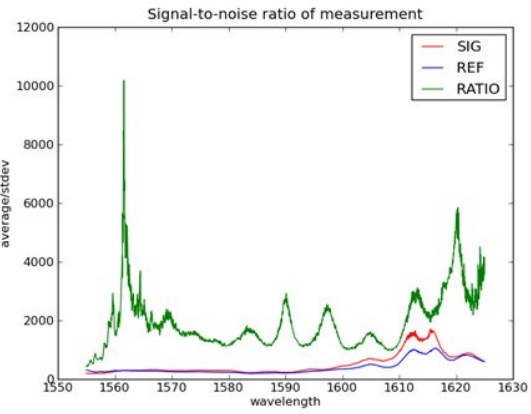

(b)

Figure 7. (a) schematic of dual-beam set-up (b) SNR improvement

\section{SILICON NITRIDE PLATFORM FOR BIO-SENSING APPLICATIONS}

\subsection{Introduction}

The wavelength range of 750-1200 $\mathrm{nm}$ is of specific interest in bio-sensing applications since it is bounded by the protein absorption at shorter visible wavelengths $(<750 \mathrm{~nm})$ and water absorption towards longer wavelength $(>1200 \mathrm{~nm})$ [24]. Furthermore in this band the photo-damage by lasers to living cells is minimal. $\mathrm{Si}_{3} \mathrm{~N}_{4}$ is a versatile HIC platform that fulfils the above requirements for its transparency at both visible and near infrared wavelengths $[25,26]$. Besides it is 
compatible with the well established CMOS process technology and has low thermo-optic coefficient making it less sensitive to ambient fluctuations than for example silicon. These advantages make $\mathrm{Si}_{3} \mathrm{~N}_{4}$ a viable candidate for low-cost photonic devices and ultimately help in realizing a lab-on-a-chip (LoC) platform operating in the visible-NIR spectral window. Towards these ends, we are developing both low loss $\mathrm{Si}_{3} \mathrm{~N}_{4}$ waveguides and efficient grating couplers (GCs), in the NIR wavelength to enable efficient surface-normal coupling from optical fibres or microscope objectives into photonic integrated components (PICs) without the need of using lenses, inverted tapers or chip facets. Furthermore, the use of grating couplers allows wafer-level testing of PICs. GCs with high efficiency have been successfully demonstrated for telecom wavelengths in many HIC material systems such as silicon [27], indium phosphide membranes, aluminum nitride [28], and $\mathrm{Si}_{3} \mathrm{~N}_{4}$ [29]. However, GCs for a HIC platform at shorter wavelengths (400-1000 nm) have not been investigated in detail. GC-based PICs will help in realizing efficient and easy-to-use integrated spectrometers and sensors. We recently reported the first demonstration of GCs at NIR $(850-950 \mathrm{~nm})$ for $\mathrm{Si}_{3} \mathrm{~N}_{4}$ wires with a best experimental efficiency of $<6 \mathrm{~dB}[30]$.

\subsection{Grating couplers in $\mathrm{Si}_{3} \mathrm{~N}_{4}$}

A $200 \mathrm{~mm}$ bare Si wafer is used as the substrate. PECVD is used to first deposit $2.4 \mu \mathrm{m}$ of $\mathrm{SiO}_{2}$ followed by $\sim 220 \mathrm{~nm}$ thick $\mathrm{Si}_{3} \mathrm{~N}_{4}$ deposition on top. After the layer deposition, the waveguide and the GCs were patterned by using $193 \mathrm{~nm}$ optical lithography and a reactive ion etch process. The waveguide was deeply etched, and GCs were defined with different etch depths by controlling the etch duration. The widths of the waveguides were in the range of $500 \pm 30 \mathrm{~nm}$. The refractive index of the $\mathrm{Si}_{3} \mathrm{~N}_{4}$ was measured to be $\sim 2.01 @ 900 \mathrm{~nm}$. The period of the grating was fixed at $630 \mathrm{~nm}$ for maximum efficiency around $900 \mathrm{~nm}$ for TE polarization and was determined using CAMFR, an eigenmode expansion tool [31]. Two types of GCs were designed - linear GC (LGC) and focusing GC (FGC). In the case of LGC, the two GCs at either end of the waveguide were defined on a $10 \mu \mathrm{m}$ wide waveguide which was adiabatically tapered down to a $500 \mathrm{~nm}$ wire. A FGC focuses the light directly from a $10 \mu$ m wide waveguide to a $450 \mathrm{~nm}$ wide wire. GC measurements were performed by coupling unpolarized light from a tungsten halogen white light source (400-1700 nm) using a singlemode fibre into the $\mathrm{Si}_{3} \mathrm{~N}_{4}$ waveguides. Another similar fibre is positioned above the output $\mathrm{GC}$ to collect the light into an optical spectrum analyzer. Both fibres were mounted on a goniometer-controlled stage to change the tilt angle. The coupling efficiency was determined from the fibre-to-fibre transmission.

The loss in the unclad $\mathrm{Si}_{3} \mathrm{~N}_{4}$ wire was estimated to be $\sim 4 \mathrm{~dB} / \mathrm{cm} @ 900 \mathrm{~nm}$. The efficiency for the GCs (LGC and FGC) as a function of wavelength for different etch depths is shown in figure 8. The efficiency is plotted for the optimum angle for different etch depths that was calculated separately by measuring transmitted power as a function of incidence angle. On comparison, $120 \mathrm{~nm}$ etched FGC exhibited best efficiency $(-5.7 \mathrm{~dB})$ whereas $140 \mathrm{~nm}$ etched LGC yielded the best 1 $\mathrm{dB}$ bandwidth $(37 \pm 2 \mathrm{~nm})$. The discrepancy between the theoretical and experimental results is attributed to the variation in the buried oxide thickness $(\sim 250 \mathrm{~nm})$ and excess scattering loss due to sidewall roughness. The overall efficiency is comparable with the standard $\mathrm{Si}$ GC used for telecom applications. It is worth mentioning here that $\operatorname{since} \mathrm{Si}_{3} \mathrm{~N}_{4}$ is a deposited material it is possible to further boost the efficiency of the $\mathrm{GC}(<3 \mathrm{~dB})$ by depositing metallic mirror or distributed Bragg reflectors underneath the grating [32].
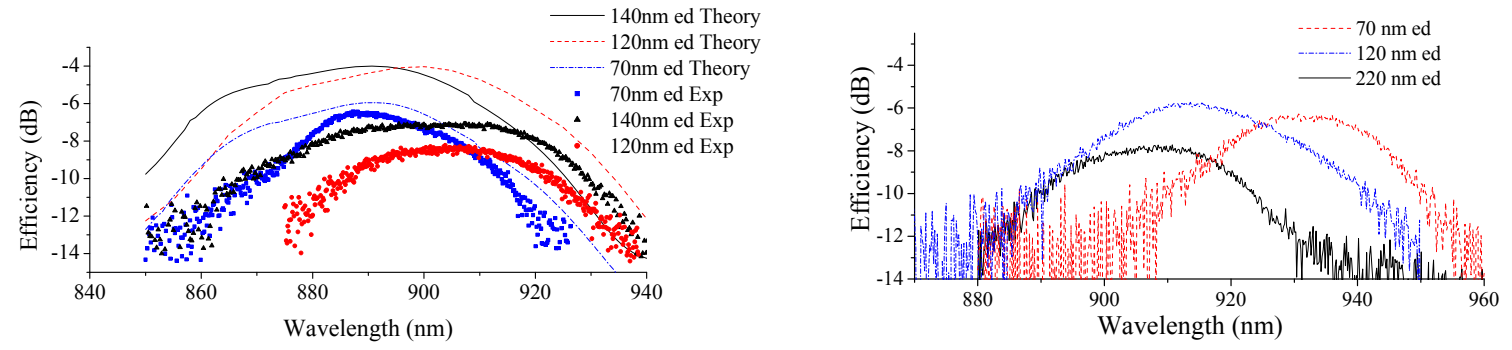

Figure 8. GC efficiency for different etch depths- LGC (left) and FGC (right). 


\section{CONCLUSION}

Spectroscopy-on-a-chip has a large potential for low-cost and miniaturized molecular sensing systems. Silicon photonics - including the use of silicon-nitride waveguides - allows to take advantage of enhancement concepts based on high refractive index contrast and on patterning with deep sub-micron accuracy. However the specific requirements for spectroscopic sensing are very different from those typically encountered in telecommunication, datacommunication and interconnect applications and are more demanding in certain aspects. Therefore there is a need for new approaches both in design and in technology so as to achieve performance levels that satisfy useful applications. In this paper, a range of developments towards that goal have been discussed.

\section{ACKNOWLEDGEMENTS}

The authors acknowledge support from the IWT-SBO Glucosens project and from the ERC-InSpectra Advanced Grant.

\section{REFERENCES}

[1] J. Hubner, J., Jorgensen A.M., Anhoj, T.A., and Zauner, D. A., "Integrated optical systems for lab-on-chip applications," Proc. SPIE 5728, 269-277 (2005).

[2] Momeni, B., Yegnanarayanan, S., Soltani, M., Eftekhar, A. A., Shah Hosseini, E., and Adibi, A., "Silicon nanophotonic devices for integrated sensing," J. Nanophoton. 3(1), 031001 (2009).

[3] Baets, R., "Building a sustainable future for silicon photonics", 8th International Conference on Group IV Photonics 2011, United Kingdom, p. 3-4 (PLE2) (2011).

[4] Bogaerts, W., Selvaraja, S., Dumon, P., Brouckaert, J., De Vos, K., Van Thourhout, D. and Baets, R., "Siliconon-Insulator Spectral Filters Fabricated with CMOS Technology”, IEEE J. Sel. Top. Quantum Electronics, 16(1), p.33-44 (2010).

[5] Selvaraja, S., Bogaerts, W., Dumon, P., Van Thourhout, D. and Baets, R., Sub-nanometer linewidth uniformity in silicon nano-photonic waveguide devices using CMOS fabrication technology , IEEE Journal on Selected Topics in Quantum Electronics, 16(1), p.316 - 324 (2010).

[6] Roelkens, R., Liu, L., Liang D., Jones R., Fang A., Koch B., Bowers J., III-V/silicon photonics for on-chip and inter-chip optical interconnects, Laser \& Photonics reviews, DOI: 10.1002/lpor.200900033 (2010).

[7] Dhakal, A., Subramaniyan A. Z., Thomas, N. L., Baets R., "The role of index contrast in the efficiency of absorption and emission of a luminescent particle near a slab waveguide", $16^{\text {th }}$ European Conference on Integrated Optics, Spain, 131 (2012).

[8] Novotny, L., “Allowed and forbidden light in near-field optics. I. A single dipolar light source,” J. Opt. Soc. Am. A, 14, 91-104 (1997).

[9] Novotny, L., and Hecht, B., "Principles of Nano-optics" CUP, Cambridge (2008).

[10]Lukosz, W., and Kunz, R. E., "Light emission by magnetic and electric dipoles close to plane interfaces", J. Opt. Soc. Am, 67, 1607-1619 (1977).

[11]Chance, R. R., Prock, A., and Silbey, R., "Molecular fluorescence and energy transfer near interfaces," in Advances in Chemical Physics, I. Prigogine and S. A. Rice, eds., 37(1-65), Wiley, New York, (1978).

[12] Purcell, E. M., Physical Review, 69, 681 (1948).

[13] Calvetti, D., Golub, G. H., Gragg, W. B., and L. Reichel, "Computation of Gauss-Kronrod Quadrature Rules." Math. Comput. 69, 1035-1052 (2000).

[14]Zayats, A. V. and Smolyaninov, I. I., "Near-field photonics: surface plasmon polaritons and localized surface plasmons," J. Opt. A: Pure Appl. Opt. 5, S16-S50 (2003). 
[15] Willets, K. A. and Van Duyne, R., "Localized Surface Plasmon Resonance Spectroscopy and Sensing," Annu. Rev. Phys. Chem. 58, 267-297 (2007).

[16] Hao, E. and Schatz, G.C., "Electromagnetic fields around silver nanoparticles and dimers," Journal of Chemical Physics 120(1), 357-366 (2004).

[17] Le Ru, E.C. and Etchegoin, P.G., "Rigorous justification of the $|E|^{4}$ enhancement factor in Surface Enhanced Raman Spectroscopy," Chemical Physics Letters 423, 63-66 (2006).

[18] Luchansky, M.S., Washburn, A.L., Martin, T.A., Iqbal, M., Gunn, L.C. and Bailey, R.C., "Characterization of the evanescent field profile and bound mass sensitivity of a label-free silicon photonic microring resonator biosensing platform," Biosensors and Bioelectronics 26, 1283-1291 (2010).

[19] Nitkowski, A., Chen, L. and Lipson, M., "Cavity-enhanced on-chip absorption spectroscopy using microring resonators," Optics Express 16, 11930-11936 (2008).

[20] Lai, W.-C., Chakravarty, S., Wang, X., Lin, C. and Chen, R.T., "On-chip methane sensing by near-IR absorption signatures in a photonic crystal slot waveguide," Optics Letters 36, 984-986 (2011).

[21] Vermeulen, D., De Koninck, Y., Li, Y., Bogaerts, W., Baets, R. and Roelkens, G., "Reflectionless grating couplers for SOI photonic integrated circuits," Optics Express, 22278-22283 (2012).

[22] Li, Y. and Baets, R., "Improved multi-mode interferometers (MMIs) on silicon-on-insulator with the optimized return loss and isolation," 16th Annual Symposium of the IEEE Photononics Benelux Chapter, Belgium (2011).

[23] Bogaerts, W., Selvaraja, S., "Compact single-mode silicon hybrid rib/strib waveguide with adiabatic bends," IEEE Photonics Journal 3(3), 422-232 (2011).

[24] Neuman, K.C. and Block, S.M., "Optical trapping,” Rev. Sci. Instrum. 75, 2787-2809 (2004).

[25] Gorin, A., Jaouad, A., Grondin, E., Aimez, V. and Charette, P., "Fabrication of silicon nitride waveguides for visible light using PECVD: a study of the effect of plasma frequency on optical properties," Opt. Exp. 18, 13509-13516 (2008).

[26] Milgram, J.N., Wojcik, J., Mascher, P. and Knights, A. P., "Optically pumped Si nanocrystal emitter integrated with low loss silicon nitride waveguides," Opt. Exp. 15, 14679-14688 (2007).

[27] Van Laere, F., Roelkens, G., Ayre, M., Schrauwen, J., Taillaert, D., Thourhout, D.V., Krauss, T.F. and Baets, R., "Compact and highly efficient grating couplers between optical fiber and nanophotonic waveguides," J. Lightw. Technol. 25, 151-156 (2007).

[28] Ghosh, S., Doerr, C.R., and Piazza, G., "Aluminum nitride grating couplers," Appl. Opt. 51, 3763-3767 (2012).

[29] Doerr, C.R., Chen, L., Chen, Y.K., and Buhl, L.L., "Wide bandwidth silicon nitride grating coupler," Photon. Technol. Lett. 22, 1461-1463 (2010).

[30] Subramanian, A.Z., Selvaraja, S., Verheyen, P., Dhakal, A., Komorowska, K., Baets, R, "Near-infrared grating couplers for silicon nitride photonic wires," Photon.Technol. Lett. 24, 1700-1703 (2012).

[31] Taillaert, D., Bienstman, P., and Baets, R., "Compact efficient broadband grating coupler for silicon-oninsulator waveguides," Opt. Lett. 29, 2749-2751 (2004).

[32] Van Laere, F., Roelkens, R., Ayre, M., Schrauwen, J., Taillaert, D., Van Thourhout, D., Krauss, T.F. and Baets, R., Compact and highly efficient grating couplers between optical fiber and nanophotonic waveguides, Journal of Lightwave Technology, 25(1), p.151-156 (2007) 